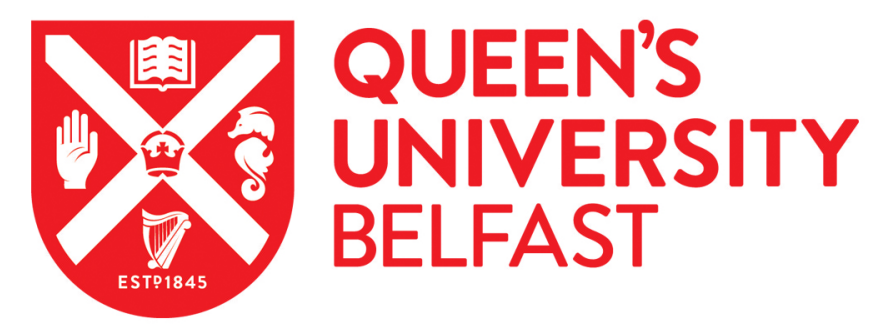

\title{
Electron paramagnetic resonance studies of the neutral nitrogen vacancy in diamond
}

Felton, S., Edmonds, A. M., Newton, M. E., Martineau, P. M., Fisher, D., \& Twitchen, D. J. (2008). Electron paramagnetic resonance studies of the neutral nitrogen vacancy in diamond. Physical Review $B$ (Condensed Matter), 77(8), [081201]. https://doi.org/10.1103/PhysRevB.77.081201

Published in:

Physical Review B (Condensed Matter)

Document Version:

Publisher's PDF, also known as Version of record

Queen's University Belfast - Research Portal:

Link to publication record in Queen's University Belfast Research Portal

Publisher rights

(C) 2008 The American Physical Society

\section{General rights}

Copyright for the publications made accessible via the Queen's University Belfast Research Portal is retained by the author(s) and / or other copyright owners and it is a condition of accessing these publications that users recognise and abide by the legal requirements associated with these rights.

Take down policy

The Research Portal is Queen's institutional repository that provides access to Queen's research output. Every effort has been made to ensure that content in the Research Portal does not infringe any person's rights, or applicable UK laws. If you discover content in the Research Portal that you believe breaches copyright or violates any law, please contact openaccess@qub.ac.uk. 


\title{
Electron paramagnetic resonance studies of the neutral nitrogen vacancy in diamond
}

\author{
S. Felton, A. M. Edmonds, and M. E. Newton* \\ Department of Physics, University of Warwick, Coventry, CV4 7AL, United Kingdom \\ P. M. Martineau and D. Fisher \\ DTC Research Centre, Belmont Road, Maidenhead, Berkshire, SL6 6JW, United Kingdom \\ D. J. Twitchen \\ Element Six Ltd., King's Ride Park, Ascot, Berkshire, SL5 8BP, United Kingdom
}

(Received 20 December 2007; published 6 February 2008)

\begin{abstract}
Despite the numerous experimental and theoretical studies on the negatively charged nitrogen vacancy center $\left(\mathrm{NV}^{-}\right)$in diamond and the predictions that the neutral nitrogen vacancy center $\left(\mathrm{NV}^{0}\right)$ should have an $S=\frac{1}{2}$ ground state, $\mathrm{NV}^{0}$ has not previously been detected by electron paramagnetic resonance (EPR). We report new EPR data on a trigonal nitrogen-containing defect in diamond with an $S=\frac{3}{2}$ excited state populated via optical excitation. Analysis of the spin Hamiltonian parameters and the wavelength dependence of the optical excitation leads to assignment of this $S=\frac{3}{2}$ state to the ${ }^{4} A_{2}$ excited state of $\mathrm{NV}^{0}$. This identification, together with an examination of the electronic structure of the NV centers in diamond, provides a plausible explanation for the lack of observation (to date) of an EPR signal from the $\mathrm{NV}^{0}$ ground state.
\end{abstract}

DOI: 10.1103/PhysRevB.77.081201

PACS number(s): 81.05.Uw, 61.72.J-, 76.30.Mi

The negatively charged nitrogen vacancy defect $\left(\mathrm{NV}^{-}\right)$in diamond is a much studied ( $>150$ papers since 2000) color center which has a zero phonon line (ZPL) at $1.945 \mathrm{eV}$ (Ref. 1) and an $S=1$ ground state with long spin coherence time. ${ }^{2}$ These properties have led to a great deal of interest in $\mathrm{NV}^{-}$, focused primarily on developing quantum information technologies (e.g., single-photon source for quantum communication $^{3}$ and as a candidate for a qubit in solid-state quantum computation $\left.{ }^{4}\right)$.

The nitrogen vacancy consists of a substitutional nitrogen impurity and a nearest-neighbor lattice vacancy to create a color center with trigonal $\left(C_{3 v}\right)$ symmetry. It can be modeled using the defect-molecule approach ${ }^{5}$ where it is assumed that the properties are determined by electrons in the dangling orbitals on the carbon and nitrogen atoms neighboring the vacancy. The $C_{3 v}$ point group requires that the three resulting linear combinations of orbitals must transform as $a_{1}, a_{1}$, and $e$. Labeling the dangling orbitals $\varphi_{\mathrm{N}}$ (for the nitrogen) and $\varphi_{a}, \varphi_{b}$, and $\varphi_{c}$ (for the three carbon neighbors) we find that

$$
\begin{gathered}
a_{1 \mathrm{~N}}=\varphi_{\mathrm{N}}, \quad a_{1 \mathrm{C}}=\frac{1}{\sqrt{3}}\left(\varphi_{a}+\varphi_{b}+\varphi_{c}\right), \\
e=\frac{1}{\sqrt{2}}\left(\varphi_{a}-\varphi_{b}\right), \quad \frac{1}{\sqrt{6}}\left(2 \varphi_{c}-\varphi_{b}-\varphi_{a}\right),
\end{gathered}
$$

where the subscripts $\mathrm{N}$ and $\mathrm{C}$ indicate the singlet levels derived from nitrogen $\left(a_{1 \mathrm{~N}}\right)$ and carbon $\left(a_{1 \mathrm{C}}\right)$ dangling orbitals, respectively. For $\mathrm{NV}^{-}$there are six electrons to be accommodated in these orbitals. Assuming that the order of these energy levels is $a_{1 \mathrm{~N}}<a_{1 \mathrm{C}}<e$, the lowest-energy configuration $a_{1 \mathrm{~N}}^{2} a_{1 \mathrm{C}}^{2} e^{2}$ gives rise to the many-electron states ${ }^{1} A_{1},{ }^{3} A_{2}$, and ${ }^{1} E$. Promoting an electron from a singlet to the doublet gives the configuration $a_{1 \mathrm{~N}}^{2} a_{1 \mathrm{C}}^{1} e^{3}$ and the many-electron states ${ }^{1} E$ and ${ }^{3} E$. Electron paramagnetic resonance (EPR) and optical studies have identified ${ }^{3} A_{2}$ as the ground state and have as- sociated the $1.945 \mathrm{eV}$ ZPL with a ${ }^{3} A_{2}$ to ${ }^{3} E$ transition. ${ }^{1,6}$ Determining the order of the energy levels is a taxing theoretical problem, and the existence of at least one metastable state between the ${ }^{3} A_{2}$ and ${ }^{3} E$ states has been proposed to explain the photophysics of $\mathrm{NV}^{-}$(Refs. 6 and 7). The details of the ${ }^{3} A_{2}$ (Refs. 8 and 9) and ${ }^{3} E$ (Ref. 9) states are still being refined, but the ground state is reasonably well understood. The small nitrogen hyperfine interaction $(A)$, the large nitrogen quadrupole interaction, and the large zero-field $(D)$ splitting (dominated by exchange coupling) in the ground state arise because the unpaired electron probability density is almost entirely on the three carbon dangling orbitals. The nitrogen is bonded to three nearest-neighbor carbons with effectively a lone pair of electrons pointing into the vacancy.

The neutral nitrogen vacancy has five electrons with which to populate the one-electron levels, and the $a_{1 \mathrm{~N}}^{2} a_{1{ }^{2}}^{2} e^{1}$ configuration gives rise to the many-electron ground state ${ }^{2} E$. Promoting an electron from a singlet to the doublet gives the configuration $a_{1 \mathrm{~N}}^{2} a_{1 \mathrm{C}}^{1} e^{2}$ and the many-electron states ${ }^{2} A_{1}$, ${ }^{4} A_{2}$, and ${ }^{2} E$. The $\mathrm{NV}^{0} \mathrm{ZPL}$ at $2.156 \mathrm{eV}$ has been shown to arise from a transition between an $E$ ground and an $A$ excited state. ${ }^{10}$ The spin of these states was not determined in the uniaxial stress-splitting study of the ZPL, but the assignment of these states to ${ }^{2} E$ (ground) and ${ }^{2} A_{1}$ follows from simple electronic structure arguments. The $S=\frac{1}{2}{ }^{2} E$ ground state has not been detected by EPR which on first inspection is surprising. One would expect an electronic Zeeman $g$ value (g) close to that of the free spin and a small hyperfine coupling (since there is no unpaired electron probability density on the nitrogen), so it is possible that the EPR spectrum is obscured by other paramagnetic defects. Overlapping spectra would make observation more difficult but it is hard to believe that this explains the lack of detection. A more likely explanation is that the dynamic Jahn-Teller distortion of the ${ }^{2} E$ ground state ${ }^{10}$ broadens the EPR lines sufficiently to preclude detection. Neither problem would apply to the ${ }^{4} A_{2}$ excited state if 
this could be populated by optical excitation. The ${ }^{4} A_{2}$ excited state is expected to be relatively low lying (i.e., below the ${ }^{2} A_{1}$ excited state), and since it is the only $S=\frac{3}{2}$ state, it is likely that it would have a sufficiently long lifetime for detection by EPR. The same argument applies to the ${ }^{5} A_{2}$ state of the neutral vacancy which has been observed in optically excited EPR experiments. ${ }^{11}$

The NV center can be produced by irradiation damage and subsequent annealing in diamond containing single substitutional nitrogen $\left(\mathrm{N}_{\mathrm{S}}\right) . \mathrm{N}_{\mathrm{S}}$ is an effective trap for vacancies, which are mobile above about $900 \mathrm{~K}^{12} \mathrm{~N}_{\mathrm{S}}^{0}$ is a deep donor, and charge transfer to $\mathrm{NV}^{0}$ produces $\mathrm{NV}^{-}$; both charge states $\mathrm{NV}^{0}$ and $\mathrm{NV}^{-}$are routinely detected by photoluminescence (PL) in the same diamond. ${ }^{2}$ Photochromism in NV centers has been reported ${ }^{13}$ and related to photoionization of other defects (e.g., $\mathrm{N}_{\mathrm{S}}^{0}$ ). However, at the excitation intensities used here no photochromism was observed. Nitrogen or $\mathrm{N}_{2}$ ion implantation can be used to produce NV centers in high-purity diamond at specified locations. ${ }^{14} \mathrm{NV}$ centers are found in as-grown nitrogen-doped diamond produced via chemical vapor deposition (CVD), ${ }^{2}$ although it is not clear if they are grown in as a unit or produced by the capture of a vacancy (mobile at typical growth temperatures) at $\mathrm{N}_{\mathrm{S}}$. High-pressure and high-temperature (HPHT) annealing of natural diamond, to remove brown coloration, ${ }^{15}$ also produces NV centers. ${ }^{15}$ It is believed that the brown coloration comes from vacancy clusters; ${ }^{16}$ vacancies released upon annealing are trapped at $\mathrm{N}_{\mathrm{S}}$ defects, possibly produced by dissociation of nitrogen aggregates, and $\mathrm{NV}$ defects are quenched in as the temperature is reduced.

The sample used in this study was produced by a modified HPHT process in which the reaction mixture (carbon source and a solvent-catalyst) in the growth capsule was pretreated at a high temperature under vacuum to remove the vast majority of the atmospheric gases. At a reduced temperature the removed gases were replaced with ${ }^{15} \mathrm{~N}$-enriched gas and the capsule was sealed. The pretreated reaction mixture was then subjected to HPHT conditions in the diamond stable region of the carbon phase diagram. ${ }^{17}$ In this way a $\mathrm{N}_{\mathrm{S}}$-doped diamond $\left(\sim 150 \mathrm{ppm} \mathrm{N} \mathrm{N}_{\mathrm{S}}^{0}\right.$, enriched with $>95 \%{ }^{15} \mathrm{~N}$, was produced. The diamond was subsequently HPHT annealed to aggregate the majority of the nitrogen $(>80 \%$ aggregation to nitrogen pairs or $A$ centers), irradiated with $1.5-\mathrm{MeV}$ electrons to a dose of $4 \times 10^{17} \mathrm{~cm}^{-2}$ and annealed at $1100 \mathrm{~K}$ in a nonoxidizing atmosphere, to form $\mathrm{NV}$ centers. $\mathrm{N}_{\mathrm{S}}^{0}$ is a much more effective trap for vacancies than the $A$ center $^{18}$ and the HPHT anneal reduced the $\mathrm{N}_{\mathrm{S}}^{0}$ concentration so that there was a high probability of producing $\mathrm{NV}^{0}$, rather than predominately $\mathrm{NV}^{-}$.

The sample contained several growth sectors, and since nitrogen uptake is strongly growth sector dependent, ${ }^{19}$ the sample was inhomogenously doped. The average bulk $\mathrm{NV}^{-}$ concentration was determined to be 0.6(1) ppm from EPR spectra recorded in the dark. Both $\mathrm{NV}^{0}$ and $\mathrm{NV}^{-}$were detected in optical absorption and PL measurements, with the absorption data suggesting that these were present in similar concentrations.

EPR spectra were recorded at $\sim 9.6 \mathrm{GHz}$ using a Bruker EMX spectrometer equipped with an Oxford Instruments liq-
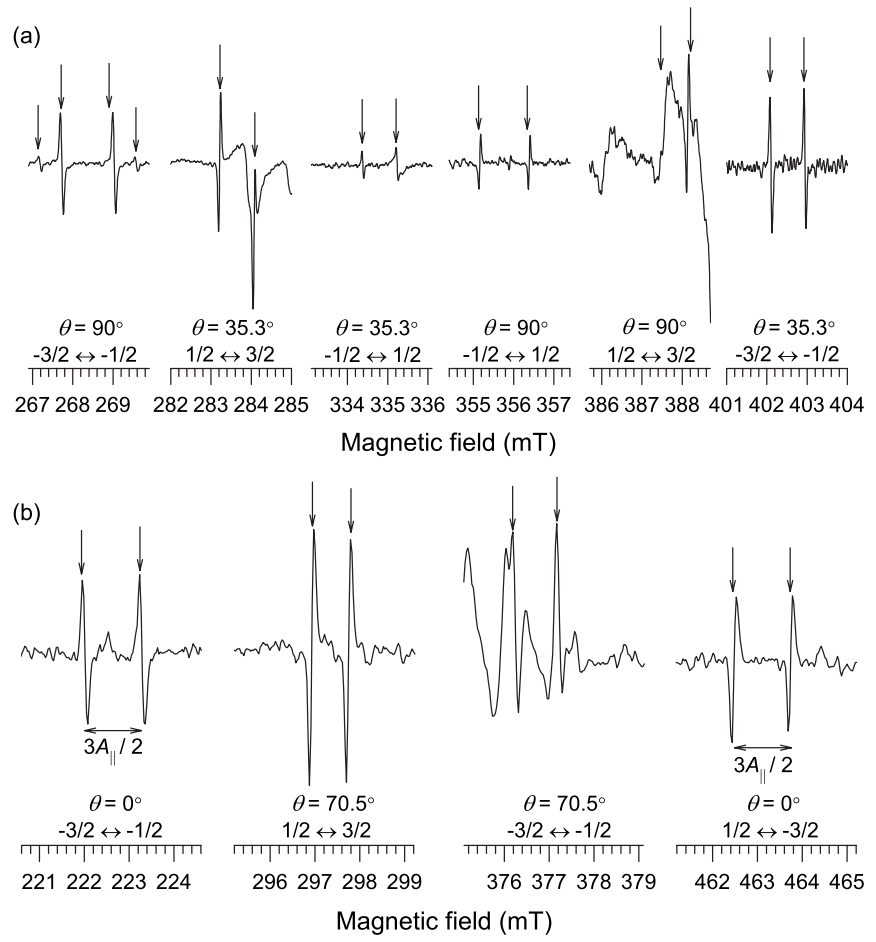

FIG. 1. EPR spectra recorded with (a) $\underline{\mathbf{B}} \|\langle 110\rangle$ at $10 \mathrm{~K}$ and (b) $\underline{\mathbf{B}} \|\langle 111\rangle$ at room temperature. The EPR transitions for the different sites are indicated by arrows and labeled with the appropriate values of $S_{z}$ (e.g., transition $S_{z}=\frac{3}{2} \rightarrow \frac{1}{2}$ ). $\theta$ denotes the angle between the defect site symmetry axis and $\underline{\mathbf{B}}$. The smaller resonances seen around the lowest-field hyperfine pair in (a) are forbidden electronnuclear double spin flips. The remaining structure originates from $\mathrm{NV}^{-}$. In (b) the ${ }^{15} \mathrm{~N}$ hyperfine splitting for the defect with principal axis parallel to the applied field is indicated; see also Table I.

uid helium ESR900 cryostat enabling measurements to be made between $4 \mathrm{~K}$ and $300 \mathrm{~K}$. Light from a $200 \mathrm{~W} \mathrm{Hg-Xe}$ arc lamp, appropriately filtered, was focused onto the sample. Figure 1(a) shows an EPR spectrum obtained with the magnetic field applied parallel to $\langle 110\rangle$ (i.e., $\mathbf{B} \|\langle 110\rangle$ ) while the sample was illuminated with the output of the lamp in the region 300-700 $\mathrm{nm}$. The spectrum shows a pattern of six pairs of lines. A trigonal defect in diamond has four symmetry-related copies orientated along [111], [ī11], $[1 \overline{1} \overline{1}]$, and $[\overline{1} 1 \overline{1}]$ so that when $\mathbf{B} \|\langle 110\rangle$ the symmetry axis of two sets of defects makes an angle of $35.26^{\circ}$ with the magnetic field and for the other two the angle is $90^{\circ}$. Thus, for an $S=\frac{3}{2}$ trigonal defect with $\underline{\mathbf{B}} \|\langle 110\rangle$, the $g$ and $D$ interactions (assumed axially symmetric about the trigonal axis) will produce two sets of three lines corresponding to the allowed EPR transitions $\left(\Delta S_{z}= \pm 1\right)$. A hyperfine coupling (also assumed axially symmetric about the trigonal axis) with an $I=\frac{1}{2}$ nucleus will split each of the six lines into two, producing the pattern of six pairs of lines observed in Fig. 1(a). Figure 1(b) shows a corresponding EPR spectrum recorded with $\mathbf{B} \|\langle 111\rangle$. In this figure only four pairs of hyperfine lines are shown, since the $S_{z}=-\frac{1}{2} \rightarrow \frac{1}{2}$ transitions are obscured by the signal from residual $\mathrm{N}_{\mathrm{S}}^{0^{2}}$.

The angular dependence of all the EPR spectra for the 
TABLE I. Spin Hamiltonian parameters for ${ }^{15} \mathrm{NV}^{0} \cdot g, D$, and $A$ have principal axes along [111].

\begin{tabular}{ccccc}
\hline \hline$g_{\|}$ & $g_{\perp}$ & $D / \mathrm{MHz}$ & $A_{\|} / \mathrm{MHz}$ & $A_{\perp} / \mathrm{MHz}$ \\
\hline $2.0029(2)$ & $2.0035(2)$ & $1685(5)$ & $-35.7(3)$ & $-23.8(3)$ \\
\hline \hline
\end{tabular}

new color center is reproduced by setting $S=\frac{3}{2}$ and $I=\frac{1}{2}$ along with constraining $g, D$, and $A$ to be axially symmetric about the trigonal axis of the defect. Spin Hamiltonian parameters for the new defect, determined from roomtemperature data, are given in Table I.

The hyperfine interaction is approximately 10 times larger than for ${ }^{15} \mathrm{NV}^{-}$(Ref. 20) and $\left|A_{\|}\right|>\left|A_{\perp}\right|$, indicating that there is a small but significant localization of the unpaired electron probability density on the nitrogen. Since the nuclear $g$ factor for ${ }^{15} \mathrm{~N}$ is -0.5664 , a negative sign is assigned to $A_{\|}$and $A_{\perp}$. This has the consequence of setting $D$ as positive, since only with $A_{\|}, A_{\perp}<0$ and $D>0$ can the position and intensity of the forbidden electron-nuclear double spin flips (see Fig. 1) be reproduced. It is also important to note that replacing ${ }^{15} \mathrm{~N}$ with another $I=\frac{1}{2}$ nucleus (e.g., $\left.{ }^{1} \mathrm{H}\right)$ does not yield a satisfactory fit to these transitions, regardless of the sign of $A$ and $D$. This unambiguously identifies ${ }^{15} \mathrm{~N}$ as the nucleus responsible for the hyperfine interaction.

The EPR spectrum for the new defect has only been observed when the sample is illuminated and, as can be seen from Fig. 1, is spin polarized. It is well known that for $\mathrm{NV}^{-}$ optical pumping causes spin polarization of the ground state $^{6,7}$ with preferential population of the $S_{z}=0$ spin sublevel, ${ }^{21}$ giving rise to a large population difference ( $\gg$ Boltzmann) between the $S_{z}=0$ and $S_{z}= \pm 1$. This results in the observation of EPR transitions in strong absorption and emission. Optical spin polarization of the ${ }^{5} A_{2}$ excited state of the neutral vacancy in diamond has also been reported. ${ }^{11}$ Figure 2 shows the variation of the room-temperature EPR intensity and hence spin polarization of the new $S=\frac{3}{2}$ color center, as well as $\mathrm{NV}^{-}$, with the energy of the exciting light (varied using low-pass filters). For $\mathrm{NV}^{-}$it is known that the optical pumping involves the ${ }^{3} A_{2}$ to ${ }^{3} E \mathrm{ZPL}$ at $1.945 \mathrm{eV}$ (Ref. 1) and selective intersystem crossing (originating from anisotropic spin-orbit coupling) to a low-lying ${ }^{1} A_{1}$ singlet. ${ }^{9}$ The threshold at 1.9(1) eV observed for spin polarization of $\mathrm{NV}^{-}$is consistent with previous work.

The threshold for observation of the spin-polarized $S=\frac{3}{2}$ system is $2.2(1) \mathrm{eV}$, with preferential (but not equal) population of the $S_{z}= \pm \frac{1}{2}$ levels with respect to the $S_{z}= \pm \frac{3}{2}$ levels. Unlike the ground state of $\mathrm{NV}^{-}$, the $S=\frac{3}{2}$ system could not be observed without optical excitation, even with extensive signal averaging; upon removal of the illumination, the signal disappeared in $\ll 1 \mathrm{~s}$. The magnitude of spin polarization did not vary strongly with temperature between $10 \mathrm{~K}$ and $300 \mathrm{~K}$. The apparent requirement of optical excitation for EPR detection of the $S=\frac{3}{2}$ system invites the assertion that this is an excited state of a defect and all the experimental evidence strongly supports the association of this system with the ${ }^{4} A_{2}$ excited state of $\mathrm{NV}^{0}$. Spin-selective intersystem crossing to the $S=\frac{3}{2}$ excited state gives rise to the observed

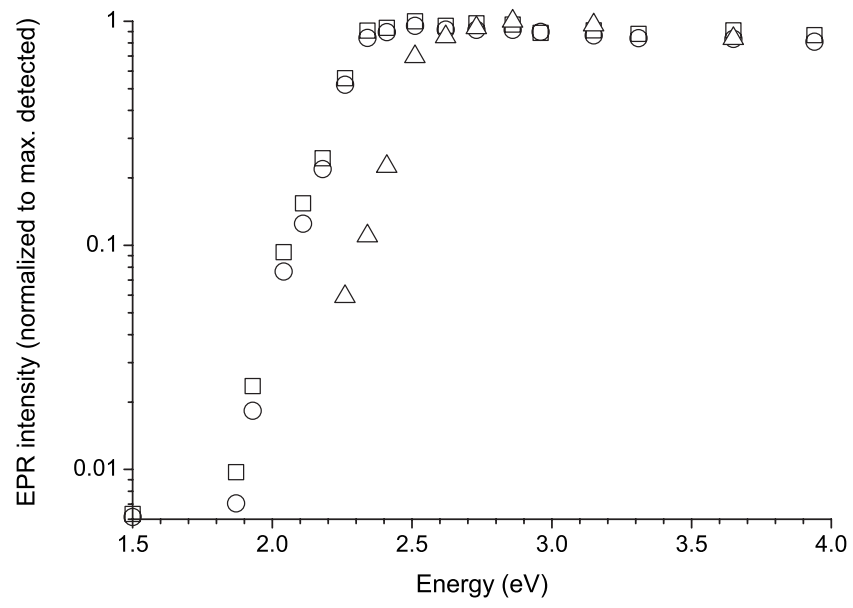

FIG. 2. Room-temperature EPR signal intensity as a function of optical excitation energy for the $S_{z}=\frac{1}{2} \rightarrow \frac{3}{2}$ transition of the $S=\frac{3}{2}$ defect $(\triangle)$ and the $S_{z}=0 \rightarrow 1(\square)$ and $S_{z}=-1 \rightarrow 0(\bigcirc)$ transitions of $\mathrm{NV}^{-}$. The data were recorded with $\mathbf{B} \|\langle 111\rangle$ for the defects with their axes parallel to the applied field. Below $2.2 \mathrm{eV}$ no EPR signal for the $S=\frac{3}{2}$ defect could be detected. The error in the cut-off energy is $\pm 0.1 \mathrm{eV}$.

spin polarization. Efficient spin polarization could increase the detection sensitivity by a factor of several hundred compared to that of a ground state in thermal equilibrium, and such a state would be below current detection limits.

There is now a considerable body of evidence supporting the assignment of the $S=\frac{3}{2}$ system to the ${ }^{4} A_{2}$ excited state of $\mathrm{NV}^{0}$. The symmetry of the EPR spin Hamiltonian parameters identifies this as a trigonal defect with a nitrogen atom on the trigonal axis. The production of the defect by irradiation damage and annealing in $\mathrm{N}_{\mathrm{S}}$-doped diamond concurrently with $\mathrm{NV}^{-}$adds further support to the assignment. The ${ }^{4} A_{2}$ state is derived from the configuration $a_{1 \mathrm{~N}}^{2} a_{1 \mathrm{C}}^{1} e^{2}$; hence, the unpaired electron probability density is expected to be predominately localized in the carbon dangling orbitals surrounding the vacancy, as is the case for $\mathrm{NV}^{-}$. A large zerofield splitting arising from exchange coupling (cannot be dipolar in origin since $D>0$ ) is therefore expected and observed. The data for the ${ }^{4} A_{2}$ excited state of $\mathrm{NV}^{0}$ indicate that approximately $6 \%$ of the unpaired electron probability density is localized on the nitrogen which is much larger than for the ${ }^{3} A_{2}$ ground state of $\mathrm{NV}^{-}$(Ref. 22) which was derived from the configuration $a_{1 \mathrm{~N}}^{2} a_{1 \mathrm{C}}^{2} e^{2}$. The properties of the ${ }^{3} A_{2}$ ground state of $\mathrm{NV}^{-}$are insensitive to the order or energy separation of $a_{1 \mathrm{C}}$ and $a_{1 \mathrm{~N}}$ levels, and the unpaired electron probability density on the nitrogen is negligible. However, for $\mathrm{NV}^{0}$, if the order of $a_{1 \mathrm{C}}$ and $a_{1 \mathrm{~N}}$ were reversed, then the configuration $a_{1 \mathrm{C}}^{2} a_{1 \mathrm{~N}}^{1} e^{2}$ would still produce the ${ }^{4} A_{2}$ excited state but now there would be significant unpaired electron probability density on the nitrogen and hence a large nitrogen hyperfine interaction. This is not likely, but it is probable that there is some mixing of the $a_{1 \mathrm{~N}}$ and $a_{1 \mathrm{C}}$ one-electron levels and this gives rise to the observed nitrogen hyperfine interaction. It is worth noting that the experimental studies were greatly simplified using ${ }^{15} \mathrm{~N}$-doped diamond; the ${ }^{14} \mathrm{~N}$ quadrupole interaction would have complicated the spectra. Measurements on similarly prepared ${ }^{14} \mathrm{~N}$-doped samples are 
planned to determine the quadrupole interaction. ${ }^{13} \mathrm{C}$ hyperfine measurements would also be useful in confirming the localization of the unpaired electron probability density, but to date these satellites have been undetectable with a $1.1 \%$ natural abundance of ${ }^{13} \mathrm{C}$.

The threshold for the optical excitation of the $S=\frac{3}{2}$ excited state matches the energy of the ZPL of $\mathrm{NV}^{0}$, providing further evidence for assignment to the ${ }^{4} A_{2}$ state. This state is expected to be low lying, with a sufficiently long lifetime (and spin lattice relaxation time) such that selective intersystem crossing could build up a spin-polarized population under conditions of optical excitation. If only $1 \%$ of the $\mathrm{NV}^{0}$ defects were excited into the ${ }^{4} A_{2}$ state, then spin polarization comparable to that measured for the $\mathrm{NV}^{-}$would produce the observed EPR intensity, assuming that $\mathrm{NV}^{-}$and $\mathrm{NV}^{0}$ are in comparable concentrations, as was indicated by the optical absorption measurements. The lack of detection of the ground state is likely to be due to dynamic Jahn-Teller coupling which broadens the EPR lines, dramatically reducing the detection sensitivity (i.e., a tenfold increase in linewidth would reduce the detection sensitivity by a factor of 100). If this is the case, then application of uniaxial stress should lock the defect into a lower-symmetry configuration, enabling EPR and optically detected magnetic resonance studies of the ground state. No donor is required for the production of $\mathrm{NV}^{0}$ (cf. $\mathrm{NV}^{-}$), simplifying its production for applications exploiting the spin physics.

The authors thank Michael Baker for helpful discussions and Joh Hansen of E6 Ltd. for synthesis of the sample. *m.e.newton@warwick.ac.uk

${ }^{1}$ G. Davies and M. F. Hamer, Proc. R. Soc. London, Ser. A 348, 285 (1976).

${ }^{2}$ T. A. Kennedy, J. S. Colton, J. E. Butler, R. C. Linares, and P. J. Doering, Appl. Phys. Lett. 83, 4190 (2003).

${ }^{3}$ F. T. Charnock and T. A. Kennedy, Phys. Rev. B 64, 041201(R) (2001).

${ }^{4}$ A. Beveratos, R. Brouri, T. Gacoin, A. Villing, J. P. Poizat, and P. Grangier, Phys. Rev. Lett. 89, 187901 (2002).

${ }^{5}$ C. Coulson and M. Kearsley, Proc. R. Soc. London, Ser. A 241, 433 (1957).

${ }^{6}$ D. A. Redman, S. Brown, R. H. Sands, and S. C. Rand, Phys. Rev. Lett. 67, 3420 (1991).

${ }^{7}$ N. R. S. Reddy, N. B. Manson, and E. R. Krausz, J. Lumin. 38, 46 (1987).

${ }^{8}$ J. P. Goss, R. Jones, S. J. Breuer, P. R. Briddon, and S. Öberg, Phys. Rev. Lett. 77, 3041 (1996).

${ }^{9}$ N. B. Manson, J. P. Harrison, and M. J. Sellars, Phys. Rev. B 74, 104303 (2006).

${ }^{10}$ G. Davies, J. Phys. C 12, 2551 (1979).

${ }^{11}$ J. A. van Wyk, O. D. Tucker, M. E. Newton, J. M. Baker, G. S. Woods, and P. Spear, Phys. Rev. B 52, 12657 (1995).

${ }^{12}$ G. Davies, S. C. Lawson, A. T. Collins, A. Mainwood, and S. J. Sharp, Phys. Rev. B 46, 13157 (1992).

${ }^{13}$ K. Iakoubovskii, G. J. Adriaenssens, and M. Nesladek, J. Phys.:
Condens. Matter 12, 189 (2000).

${ }^{14}$ J. Meijer, B. Burchard, M. Domhan, C. Wittmann, T. Gaebel, I. Popa, F. Jelezko, and J. Wrachtrup, Appl. Phys. Lett. 87, 261909 (2005).

${ }^{15}$ A. T. Collins, H. Kanda, and H. Kitawaki, Diamond Relat. Mater. 9, 113 (2000).

${ }^{16}$ L. S. Hounsome, R. Jones, P. M. Martineau, D. Fisher, M. J. Shaw, P. R. Briddon, and S. Öberg, Phys. Status Solidi C 4, 2950 (2007).

${ }^{17}$ C. V. H. Stroemann, F. Tshisikhawe, J. O. Hansen, and R. C. Burns, International Patent Application WO2006061672, 15 June 2006.

${ }^{18}$ K. Iakoubovskii and G. J. Adriaenssens, J. Phys.: Condens. Matter 13, 6015 (2001).

${ }^{19}$ R. C. Burns, V. Cvetkovic, C. N. Dodge, D. J. F. Evans, M. L. T. Rooney, P. M. Spear, and C. M. Welbourn, J. Cryst. Growth 104, 257 (1990).

${ }^{20}$ J. R. Rabeau, P. Reichart, G. Tamanyan, D. N. Jamieson, S. Prawer, F. Jelezko, T. Gaebel, I. Popa, M. Domhan, and J. Wrachtrup, Appl. Phys. Lett. 88, 134104 (2006).

${ }^{21}$ J. Harrison, M. J. Sellars, and N. B. Manson, J. Lumin. 107, 245 (2004).

${ }^{22}$ X. F. He, N. B. Manson, and P. T. H. Fisk, Phys. Rev. B 47, 8816 (1993). 